\title{
Determination of Photon and Elementary Particles Rest Masses Using Maxwell's Equations and Generalized Potential Dependent Special Relativity
}

\author{
Abeer Mohammed Khairy Ahmed ${ }^{1}$, Mashair Ahmed Mohammed Yousif', \\ Zainab Mustapha Kurawa ${ }^{3}$, Zoalnoon Ahmed Abeid Allah Saad ${ }^{4}$, Suhair Salih Makawy ${ }^{5}$, \\ Mohammed Idriss Mohammed6, Mubarak Dirar Abd-Alla6 ${ }^{6}$, Sami Abdalla Elbadawi Mohamed7 \\ ${ }^{1}$ Departmentof Physics, Faculty of Art and Science at AL Muznab, AL Qassim University, AL Muznab, Kingdom of \\ Saudi Arabia; ${ }^{2}$ Department of Physics, Faculty of Science and Education at Alkhurma, Taif University, Alkhurma, \\ Kingdom of Saudi Arabia; ${ }^{3}$ Department of Physics, School of Science Education, Saadatu Rimi College of Education, \\ Kumbosto Kano, Nigeria; ${ }^{4}$ Departmentof Physics, Faculty of Arts and Sciences, Dhahran Aljanoub, King Khalid \\ University, Dahran, Kingdom of Saudi Arabia; ${ }^{5}$ Department of Physics, Faculty of Science, Taif University, Taif, \\ Kingdom of Saudi Arabia; ${ }^{6}$ Department of Physics, Faculty of Science, Sudan University of Science and Technology, \\ Khartoum, Sudan; ${ }^{7}$ Department of Physics, Faculty of Education, Gadarif University, Gadarif, Sudan
}

Correspondence to: Mubarak Dirar Abd-Alla, mubarakdirar@gmail.com

Keywords: Photon, Elementary Particles, Maxwell's Equations, Potential Dependent Special Relativity, Vacuum Energy

Received: May 16, $2020 \quad$ Accepted: August 14, $2020 \quad$ Published: August 17, 2020

Copyright (c) 2020 by author(s) and Scientific Research Publishing Inc.

This work is licensed under the Creative Commons Attribution International License (CC BY 4.0).

http://creativecommons.org/licenses/by/4.0/

\section{(c) (i) Open Access}

\section{ABSTRACT}

The nature and origin of the photon and elementary rest masses are some of the challenging problems that physics face. The approaches used to solve these problems are complex and time-consuming. Specifically, the photon rest mass pays attention to theoretical physicists. Many experimental works show that the photon rest mass is non zero. This problem can be solved using generalized potential dependent special relativity, which has been derived using simple arguments, and Maxwell's equations, besides the conventional Einstein energy-momentum relation. The results obtained show that the rest mass of photons and elementary particles are strongly dependent on the vacuum energy and a universal constant. This result conforms with the models that predict time decaying vacuum energy associated with production of smaller rest mass particles followed by larger masses. The two potential dependent mass expressions conform with the cosmological models that suggest the photon is generated first by assuming the universe consisting of total constant vacuum with decaying cosmological part and mass generating part. Using Maxwell's equations, beside plank and De Broglie hypothesis together with special relativity energy-momentum relation the photon rest mass is estimated. It was shown that the photon rest mass is extremely small 


\section{compared to the electron mass.}

\section{INTRODUCTION}

Electromagnetic waves (EM) are described by using Maxwell's equations (ME). These equations successfully describe a wide variety of physical phenomena concerning the behavior of light as waves. These include reflection, refraction, transmission and generation of EM waves [1].

Despite these remarkable successes of ME, they fail to describe the so-called black body radiation. This forces plank to propose that EM waves (EMW) to behave as discrete isolated energy packets called quanta. According to plank hypothesis, the energy of each quanta is proportional to the frequency of the EMW [2].

This particle theory of EMW succeeded in describing many physical phenomena including photo electric effect, Compton effect and pair production [3].

This discovery of the dual nature of light and electromagnetic waves leads to propose, by De Broglie, that also atomic particles like electrons, protons and neutrons have also dual nature, in the sense that they behave as waves. These waves are called matter waves [4].

This dual nature of atomic and sub-atomic particles leads sum scientists to formulate the so-called quantum physics, by Schrodinger, Heisenberg, Klein-Garden, Dirac and others [4].

Recently the quantum laws were developed to describe the nature of fields and to unify them besides describing the nature of elementary particles $[5,6]$.

Different attempts and models were constructed to describe the behavior of fields and elementary particles. These models include quantum field theory, electroweak theory, grand unified theories and string theories $[7,8]$.

These theories succeeded in describing many phenomena associated with the behavior of elementary particles and fields. But unfortunately, these theories are complex and are incapable of describing many phenomena like the mass of neutrino and the photon besides quantizing and unifying the gravitational field with other fields $[9,10]$.

Different attempts were made to cure some of these effects by suggesting simple elegant model, by M.Dirar and others. These models are based on generalized special relativity (GSR) or potential dependent special relativity (PSR). These models explain the origin of masses of elementary particles and the high speed of neutrino, besides change of neutrino mass [11-13]. Many papers exhibit experiments done to show that the photon rest mass is non zero. Some of these papers are shown in Section 2. Sections $3 \& 4$ are devoted to construct theoretical models based on generalized special relativity (gsr) and Maxwell's equations to derive a useful expression of the photon and elementary rest masses together with the discussion.

\section{PHYSICAL AND EXPERIMENTAL EVIDENCES CONFIRMING THE NONZERO PHOTON REST MASS}

The very beginning of the hypothesis that claims zero photon rest mass from the expressions of the mass and energy in special relativity (sr). According to sr the photon mass $m$ for a particle moving with speed $v$ is given by

$$
m=\frac{m_{0}}{\sqrt{1-v^{2} / c^{2}}}
$$

where $m_{0}$ is the rest mass. Since the photon speed is

$$
v=c
$$

This means that the photon mass when moving with speed is

$$
m=\frac{m_{0}}{0}=\infty
$$


which is in direct conflict with the fact that the mass of any physical quantity is finite. It is also disagree with the plank hypo this that the photon energy $E$ for a frequency $w$ is given by

$$
E=\hbar w=m c^{2}=\frac{m_{0} c^{2}}{\sqrt{1-v^{2} / c^{2}}}
$$

where $h$ stands for Plank constant and $E$ is finite. To avoid this conflict sr suggests that the photon rest vanishes, i.e.

$$
m_{0}=0
$$

Thus from (2) and (3)

$$
\begin{aligned}
& m=\frac{0}{0}=\text { finite } \\
& E=\frac{0}{0}=\text { finite }
\end{aligned}
$$

But the suggestion that any particle has zero rest mass is not also physically acceptable. This is since it means that such particle does not exist at all. It is also unconceivable and unlogical and even unbelievable and unlogical and even unbelievable to suggest that non-existing zero rest mass particle can generate massive particle when it moves with speed. How can one believe that nothing generates something this suggestion is in direct conflict with the observed deflection of light photons by the sun which is one of the key predictions of general relativity (gr). This means that there is something wrong with sr. this can be easily discovered when comparing sr with Newton's laws, quantum mechanics and even gr. According to the energy expression (3) for energy, the energy of two particles one move in a free space and the other inside the earth gravitational field is the same, as for as they have the same speed. This does not agree with newton's laws and observations, which show that the one inside the gravitational field a quires additional potential energy. In the Newtonian limit Equation (3) gives

$$
E=m_{0} c^{2}\left(1-\frac{v^{2}}{c^{2}}\right)^{-\frac{1}{2}}=m_{0} c^{2}+\frac{1}{2} m_{0} v^{2}
$$

Unfortunately this expression (6) does not reduce to the Newtonian one

$$
E=\frac{1}{2} m_{0} v^{2}+V
$$

when the rest mass energy is neglected. Thus sr energy relation does not satisfy comes pendency principle due to the absence of potential term $V$.

Moreover sr does not conform with the effective mass notion in solid state physics, which stated that the electron mass $m^{*}$ inside the crystal is affected by both lattice crystal force $F_{i}$ as well as the external force $F_{e}$ according to the relation

$$
m^{*}=\frac{m F_{e}}{F_{e}+F_{i}}
$$

where $\mathrm{m}$ is the electron rest mass. This experimentally verified relation (8) does not conform with sr mass relation (1), which does not recognize the effect of the force the mass is affected by the speed only.

The expression of the energy (3) in sr is in direct conflict with quantum laws which show that the particle quantum energy is highly affected by the potential. According to Schrodinger equation, the energy $E$ of a particle in a box of length $L$, when no potential exist is given by

$$
E \sim \frac{h^{2} n^{2}}{2 m L^{2}}
$$


where $n$ is the principal quantum number. However for the harmonic oscillator where the potential is

$$
V \sim \frac{1}{2} k x^{2}
$$

with $k$ representing stiffness, and $x$ is the displacement the energy, due to Schrodinger equation is given by

$$
E \sim\left(n+\frac{1}{2}\right) \hbar w
$$

For spherical nucleus having $Z$ protons the electron potential $V$ satisfies

$$
V \sim \frac{Z}{r^{2}}
$$

where, $r$ is the electron position with respect to the nucleus. In this case he quantum electron energy $E$, satisfies

$$
E \sim \frac{Z^{2}}{n^{2}}
$$

$n$ is the principal quantum number in Equation (12) and Equation (14) also.

Thus it is clear that the quantum energy is highly dependent are confirmed with the observed atomic and matter spectra. This means does not incorporate potential energy is in direct conflict with quantum laws and observations.

There are also many experimental evidences which show that the photon has non zero rest mass. One of the recent papers of Mahindra and Ramesh shows that the photon rest mass depends on the curvature of the space. This means that the photon rest mass is dependent on the gravitational potential. This paper exhibit recent experiments which show that the photon rest mass has order of magnitude $10^{-54} \mathrm{~kg}$. The satellite measurement of the earth magnetic field indicates that the photon rest mass is about $1.5 \times 10^{-54} \mathrm{~kg}$. in the frequency dependent time delays in the dispersion measurements of fast radio burst, the photon rest mass is found to be $3 \times 10^{-50} \mathrm{~kg}$. the photo rest mass is found to be wave length dependent and is imaginary within or inside matter [14].

Astronomical observations enables estimating the photon rest mass as pointed out in the work exhibited in Jun-Jie and Xue-Feing paper [15]. The frequency dependent time delays of radio emission from 22 radio pulsars in the large Magellan cloud and 5 pulsars in the small one shows the photon rest mass upper bound having order of magnitude $10^{-48} \mathrm{~kg}$ [16]. Also, Narendra proved in his seminal paper that the path of the red and green Laser light show slight deviation after reflection. This means that the photon creates a force at the contact point of reflection. This indicates that the photon has non zero rest mass.

\section{POTENTIAL DEPENDENT SPECIAL RELATIVITY AND EFFECTIVE MASS AND DISCUSSION}

The potential, kinetic energy relation for constant mass particle car be obtained from the definition of the work done by a force per unit mass $F_{0}$ generating potential per unit mass $\varphi$. For general force $F$ and potential $V$, one gets

$$
\begin{gathered}
\int F \cdot \mathrm{d} r=-\int \nabla V \cdot \underline{\mathrm{d} r} \\
\int m \frac{\mathrm{d} v}{\mathrm{~d} t} \mathrm{~d} r=-\int \mathrm{d} v \\
\int m \frac{\mathrm{d} r}{\mathrm{~d} t} \mathrm{~d} v=-\int m \mathrm{~d} \varphi
\end{gathered}
$$

For unit mass $(m=i)$, thus 


$$
\begin{gathered}
\int_{v_{0}}^{v} v \mathrm{~d} v=-\int_{\varphi}^{0} \mathrm{~d} \varphi \\
{\left[\frac{1}{2} v^{2}\right]_{v_{0}}^{v}=-[\varphi]_{\varphi}^{0}} \\
\frac{1}{2} v^{2}-\frac{1}{2} v_{0}^{2}=-[0-\varphi] \\
\frac{1}{2} v^{2}=\frac{1}{2} v_{0}^{2}+\varphi \\
\frac{v^{2}=v_{0}^{2}+2 \varphi}{v_{0}^{2}=v^{2}-2 \varphi}
\end{gathered}
$$

Using the expression of the rest mass in sr

$$
m=\frac{m_{0}}{\sqrt{1-\frac{v_{0}^{2}}{c^{2}}}}
$$

A direct substitution of Equation (16) in Equation (17) gives

$$
m=\frac{m_{0}}{\sqrt{1+\frac{2 \varphi}{c^{2}}-\frac{v^{2}}{c^{2}}}}
$$

This relation can be utilized to find the effective mass relation. Neglecting the velocity effect $(v=0)$ and considering external potential $\varphi_{e}$ and the crystal lattice potentials $\varphi_{i}$ to be weak the mass and the effective mass $m^{*}$ inside the crystal are given by:

$$
\begin{gathered}
m=\frac{m_{0}}{\left(1+\frac{2 \varphi_{e}}{c^{2}}\right)^{\frac{1}{2}}}=\frac{m_{0}}{\left(\frac{m_{0} c^{2}+V_{e}}{m_{0} c^{2}}\right)} \\
m^{*}=\frac{m_{0}}{\left(1+\frac{2 \varphi}{c^{2}}\right)^{\frac{1}{2}}}=\frac{m_{0}}{\left(1+\frac{\varphi}{c^{2}}\right)}=\frac{m_{0}}{\left(\frac{m_{0} c^{2}+V}{m_{0} c^{2}}\right)}=\frac{m_{0}}{\left(\frac{m_{0} c^{2}+V_{e}+V_{i}}{m_{0} c^{2}}\right)}
\end{gathered}
$$

Hence, neglecting the rest mass energy

$$
m^{*}=\frac{m\left(m_{0} c^{2}+V_{e}\right)}{m_{0} c^{2}+V_{e}+V_{i}}=\frac{m V_{e}}{V_{e}+V_{i}}=\frac{m V_{e} L}{\left(F_{e}+F_{i}\right) L}=\frac{m F_{e}}{F_{e}+F_{i}}
$$

where for uniform forces

$$
V_{e}=F_{e} L, \quad V_{i}=F_{i} L
$$

Thus one gets the conventional effective mass relation. This means that the potential dependent special relativity or the generalized special relativity is more successful than the sr, since it reduces to sr in the absence of the field and it gives a useful expression of the effective mass inside the crystal typical to the conventional one which agrees with experimental results.

\section{PARTICLES REST MASSES USING GENERALIZED SPECIAL RELATIVITY BESIDE MAXWELL'S EQUATION AND DISCUSSION}

The rest mass of particles can be obtained by using the GSR expression for the mass moving with speed in a potential, where [12]. 


$$
m=\frac{c_{0}\left(1+2 \frac{\left(\varphi+\varphi_{0}\right)}{c^{2}}\right)}{\sqrt{1+2 \frac{\left(\varphi+\varphi_{0}\right)}{c^{2}}-\frac{v^{2}}{c^{2}}}}
$$

For a particle in vacuum $\varphi_{0}$

$$
\varphi=0, \quad v=0
$$

where the particle is at rest with no external field, and $c_{0}$ is a universal constant. Thus the rest mass mo is given by

$$
m_{0}=\frac{c_{0}\left(1+2 \frac{\varphi_{0}}{c^{2}}\right)}{\sqrt{1+2 \frac{\varphi_{0}}{c^{2}}}}=c_{0}\left(1+2 \frac{\varphi_{0}}{c^{2}}\right)^{\frac{1}{2}}
$$

This means that the rest mass mo is dependent on the constant parameter co as well as.

For a photon having angular frequency $w$, plank hypothesis and Einstein energy relations give

$$
h w=\frac{c_{0} c^{2}\left[\left(1+\frac{2 \varphi_{0}}{c^{2}}\right)+\left(\frac{2 \varphi}{c^{2}}\right)\right]}{\sqrt{1+\frac{2\left(\varphi+\varphi_{0}\right)}{c^{2}}-\frac{v^{2}}{c^{2}}}}=m c^{2}
$$

Since the photon moves with speed and since in vacuum, no potential exist, thus

$$
\begin{gathered}
\varphi=0, \quad v=0 \\
\hbar w=\frac{c_{0} c^{2}\left[1+\frac{2 \varphi_{0}}{c^{2}}\right]}{\sqrt{1+\frac{2 \varphi_{0}}{c^{2}}-1}}=\frac{c_{0} c^{2}\left[1+\frac{2 \varphi_{0}}{c^{2}}\right]}{\sqrt{\frac{2 \varphi_{0}}{c^{2}}}}
\end{gathered}
$$

But according to Equations (3), (23) and (26).

Thus the photon rest mass is given by

$$
\hbar w=\frac{m_{0} c^{2} \sqrt{1+\frac{2 \varphi_{0}}{c^{2}}}}{\sqrt{\frac{2 \varphi_{0}}{c^{2}}}}
$$

$$
m_{0}=\frac{\hbar w \sqrt{\frac{2 \varphi_{0}}{c^{2}}}}{c^{2} \sqrt{1+\frac{2 \varphi_{0}}{c^{2}}}}
$$

However when another expression of $m$ is used, which is also derived on the basis of GSR, one gets

$$
m=\frac{c_{0}}{\sqrt{1+\frac{2\left(\varphi+\varphi_{0}\right)}{c^{2}}-\frac{v^{2}}{c^{2}}}}
$$

Thus for a particle at rest $(v=0)$ in vacuum $(\varphi=0)$ one gets the rest mass to be 


$$
m_{0}=\frac{c_{0}}{\sqrt{1+\frac{2 \varphi_{0}}{c^{2}}}}
$$

However, where the photon is at rest then its energy according to Einstein energy relation is

$$
E=m_{0} c^{2}=\frac{c_{0} c^{2}\left(1+\frac{2 \varphi_{0}}{c^{2}}\right)}{\sqrt{1+\frac{2 \varphi_{0}}{c^{2}}}}
$$

Thus

$$
m_{0}=\frac{c_{0}\left(1+\frac{2 \varphi_{0}}{c^{2}}\right)}{\sqrt{1+\frac{2 \varphi_{0}}{c^{2}}}}=c_{0}\left(1+2 \frac{\varphi_{0}}{c^{2}}\right)^{\frac{1}{2}}
$$

which resembles Equation (23).

The plank hypothesis suggests that

$$
E=\hbar w_{0}=m_{0} c^{2}
$$

This means that

$$
m_{0}=\frac{c_{0}\left(1+\frac{2 \varphi_{0}}{c^{2}}\right)}{\sqrt{1+\frac{2 \varphi_{0}}{c^{2}}}}=c_{0}\left(1+2 \frac{\varphi_{0}}{c^{2}}\right)^{\frac{1}{2}}
$$

The relation between the mass for a moving body that exist in a certain field and the rest mass can be found by using Equation (23) and Equation (29) to get

$$
m=\frac{m_{0}\left(1+\frac{2 \varphi_{0}}{c^{2}}\right)^{-\frac{1}{2}}}{\sqrt{1+\frac{2\left(\varphi+\varphi_{0}\right)}{c^{2}}-\frac{v^{2}}{c^{2}}}}=\frac{m_{0}}{\sqrt{\left(1+\frac{2 \varphi_{0}}{c^{2}}\right)\left(1+\frac{2\left(\varphi+\varphi_{0}\right)}{c^{2}}-\frac{v^{2}}{c^{2}}\right)}}
$$

But one needs to answer the question, what term is responsible for making the masses of the elementary particles different from each other. According to Equation (28) and Equation (34), the rest mass of different particles were generated at different stages of the early universe, which are characterized by different values of the vacuum energy. Many models that describe the early universe suggests that vacuum energy is very large at early time then decreases gradually with time in view of Equation (30) this means that very small rest masses, like that of photons, were generated first, followed by larger masses up to very large massive particles. Fortunately, this regime conforms with that suggested by most of the successful cosmological models, including the big bang model. However Equation (33) and Equation (34) give different results, because the rest mass here increases with vacuum energy. This means that in the very early universe the heavy massive elementary particles were generated first followed by lighter ones. This view does not conform with most of the cosmological and the elementary particle models. To remove this conflict, one can assume the universe consists of cosmic and matter vacuum energies, $\varphi_{c}$ and $\varphi_{0}$, respectively, such that their sum is constant, i.e. 


$$
\varphi_{c}+\varphi_{0}=\varphi_{v}
$$

Thus when comic vacuum energy $\varphi_{c}$ is very large at early universe the matter vacuum $\varphi_{0}$ is very small, thus according to Equation (34) the photon was generated first followed by heavier particles, which conform with the cosmological models. This view can be also used to explain why photon is generated first for large comic vacuum according to Equation (30). This can be explained by suggesting that the total energy of the universe $E u$ is a sum of cosmic vacuum energy $\varphi_{0}$ and total matter energy $M c^{2}$, i.e. $\left(\varphi_{m}=M c^{2}\right)$

$$
E u=\varphi_{0}+\varphi_{m}=E u=\varphi_{0}+M c^{2}=\varphi_{0}+N m_{0} c^{2}=\text { constant }
$$

$N=$ number of particles.

Thus for very large vacuum energy, the energy available to elementary particles becomes extremely small. Thus the photon was generated first followed by heavier ones. Thus Equation (30) reads

$$
m_{0}=\frac{c_{0}}{\sqrt{1+\frac{2\left(E_{u}-\varphi_{m}\right)}{c^{2}}}}
$$

Thus one can view elementary particles as hollow spheres filled with matter vacuum $\varphi_{m}$. Thus for very large vacuum energy $\varphi_{0}$ small matter. Matter vacuum, $\varphi_{m}$ is small thus fill this sphere with matter vacuum $\varphi_{m}$ is small, thus full this hollow sphere with small matter energy. Thus it gain very small mass. The expression (1) for mass together with Maxwell's equations can be used to find the photon rest mass. The evolution of the electric field intensity $E$ for polarizing medium of polarization $p$, can be found, where

$$
p=e x
$$

with $e$ standing for electron charge, while $x$ is stands for the displacement of the electron. This evolution takes the form

$$
-\nabla^{2} E+\frac{1}{c^{2}} \frac{\partial^{2} E}{\partial t^{2}}+m_{0} \frac{\mathrm{d}^{2} P}{\mathrm{~d} t^{2}}=0
$$

The polarization term can be simplified by using the equation of motion of the electron due to the effect of electric field in vacuum, which takes the form

$$
m \ddot{x}=e E
$$

Thus

$$
\ddot{x}=\frac{e}{m} E
$$

In view of Equation (1)

$$
\begin{gathered}
\frac{\mathrm{d}^{2} P}{\mathrm{~d} t^{2}}=\left(e^{2} / m\right) E \\
-\nabla^{2} E+\frac{1}{c^{2}} \frac{\partial^{2} E}{\partial t^{2}}+\frac{e^{2} m_{0}}{m} E=0
\end{gathered}
$$

In vacuum, the electric field intensity behave as a travelling wave, in the form

$$
E=E_{0} \mathrm{e}^{i(k r-w t)}
$$

A direct differentiation with respect to $r$ and $t$ twice gives

$$
\begin{aligned}
& \nabla^{2} E=-K^{2} E \\
& \frac{\partial^{2} E}{\partial t^{2}}=-w^{2} E
\end{aligned}
$$


A direct substitution of Equation (44) in Equation (42) gives

$$
\begin{gathered}
c^{2} K^{2} E-w^{2} E+\frac{c^{2} \mu_{0} \varepsilon_{0}}{\varepsilon_{0}} \frac{e^{2}}{m} E=0 \\
c^{2} K^{2}-w^{2}+\frac{c^{2} \mu_{0} \varepsilon_{0}}{\varepsilon_{0}} \frac{e^{2}}{m}=0
\end{gathered}
$$

but

$$
c^{2}=\left(\mu_{0} \varepsilon_{0}\right)^{-1}
$$

Using also Plank and De Brogglie hypothesis photon energy and momentum are given to be

$$
\begin{aligned}
& E=\hbar w \\
& P=\hbar K
\end{aligned}
$$

And multiplying both sides of Equation (45) by $h$, yields

$$
c^{2} P^{2}-E^{2}+\frac{e^{2} \hbar^{2}}{m \varepsilon_{0}}=0
$$

Thus

$$
E^{2}=c^{2} P^{2}+\frac{e^{2} \hbar^{2}}{m \varepsilon_{0}}
$$

Comparing this with the Einstein energy momentum relation

$$
E^{2}=c^{2} P^{2}+m_{0}^{2} c^{4}
$$

Gives

$$
m_{0} c^{2}=e\left(m \varepsilon_{0}\right)^{-\frac{1}{2}} \sqrt{\hbar^{2}}
$$

Thus the photon rest mass is given by

$$
m_{0}=\frac{e}{c^{2}}\left(m \varepsilon_{0}\right)^{-\frac{1}{2}} \sqrt{\hbar^{2}}=\frac{e \hbar}{c^{2}}\left(m \varepsilon_{0}\right)^{-\frac{1}{2}}
$$

A direct substitution of the following constants

$$
\begin{gathered}
e=1.6 \times 10^{-19}=16 \times 10^{-20} \\
m=9.1 \times 10^{-31}=91 \times 10^{-32} \\
\hbar=\frac{6.6 \times 10^{-34}}{6.28}=\frac{66 \times 10^{-33}}{628} \\
c=3 \times 10^{8} \\
\varepsilon_{0}=8.85 \times 10^{-12}=885 \times 10^{-14}
\end{gathered}
$$

In Equation (33) yields

$$
\begin{gathered}
m_{0}=\frac{91 \times 10^{-32}}{9 \times 10^{16}} \times \frac{66 \times 10^{-33}}{628} \frac{1}{\left(91 \times 10^{-32} \times 885 \times 10^{-14}\right)^{\frac{1}{2}}} \\
m_{0}=10^{47} \times 10^{33} \times 10^{23} \times \frac{0.11}{283.8}=10^{-59} \times 0.039
\end{gathered}
$$




$$
m_{0}=39 \times 10^{-62} \mathrm{~kg}
$$

This indicates that the photon mass is very small and much smaller than the electron mass which is quite reasonable.

\section{CONCLUSIONS}

The experimental work done using laser and astronomical observations show that the photon aquires non zero rest mass. Using sr beside Newton's laws gsr mass expression is derived. This gs mass expression is used to derive the ordinary conventional effective mass relationships which agree with experimental observations. A useful expression of elementary particles masses based on the GPSR was obtained in terms of mass vacuum energy.

Here the total vacuum energy is assumed to be consisting of cosmological time decaying part and a mass generating growing part.

Assuming elementary particles as resulting from one hollow sphere, at very early time of the universe, the very small matter vacuum fills this sphere producing the photon first followed by larger mass elementary particles. This version conforms with that proposed by most of the cosmological models.

This mass expression when no field exists together with Maxwell's equations is used to determine the photon mass numerically. This photon mass is shown to be extremely small compared to the electron mass which agrees with observations.

\section{CONFLICTS OF INTEREST}

The authors declare no conflicts of interest regarding the publication of this paper.

\section{REFERENCES}

1. Serway, R.A. (1996) Physics. Saunders College Publishing, Philadelphia.

2. Mehta, N. (2011) Applied Physics for Engineers. PHI Learning Private Limited, New Delhi.

3. Dirac, P.A.M. (2011) The Principles of Quantum Mechanics. Oxford University Press, Oxford.

4. Mathew, P.M. and Venkatesan, K. (2007) A Text Book of Quantum Mexhanics. Tata McGraw Hill, New Delhi.

5. Lancaster, T. and Blundell, S.J. (2014) Quantum Field Theory. Oxford University Press, Oxford.

6. Lahiri, A. and Pal, P.B. (2015) A First Book of Quantum Field Theory. Narosa Publishing House, New Delhi.

7. Burcham, W.E. and Jobes, M. (1995) Nuclear and Particle Physics. Longman Scientific \& Technical, Harlow. https://doi.org/10.1063/1.2808262

8. Halzen, F. and Martin, A.D. (1984) Quarks and Leptons: An Introductions Course in Modern Physics. John Wiley, New York.

9. Zwiebach, B. (2004) A First Course in String Theory. Cambridge University Press, New York. https://doi.org/10.1017/CBO9780511841682

10. Becker, K., Becker, M. and Schwarz, J. (2007) String Theory and M-Theory: A Modern Introduction. Ambridge University Press, New York. https://doi.org/10.1017/CBO9780511816086

11. Mohammed, S.D., Dirar, M., Ibrahim, H.H. and Sawsan, A.E. (2018) Generation of Elementary Particles inside Black Holes at Plank Time. International Journal of Innovative Science, Engineering \& Technology, 5, 40-47.

12. Hillo, M.H.M. (2011) Using of the Generalized Special Relativity in Estimating the Neutrino Masses to Explain the Conversion of Electron Neutrions. Natural Science, 3, 334-338.

13. Kaku, M. (2000) Strings, Conformal Fields and M-Theory. Springer Verlag, New York. https://doi.org/10.1007/978-1-4612-0503-6 
14. Goray, M. and Annavarapu, R.N. (2020) Rest Mass of Photon on the Surface of Matter. Results in Physics, 16, Article ID: 102866. https://doi.org/10.1016/j.rinp.2019.102866

15. Wei, J.-J. and Wu, X.-F. (2018) Robust Limits on Photon Mass from Statistical Samples of Extra Galactic Radio Pulsars. Journal of Cosmology and Astroparticle Physics, 7, 45.

16. Agarwal, N.S. (2018) Experimental Proof of Massive Photon. Journal of Modern Physics, 6, 627-633. 